\title{
Displacement Correction Scheme for MR-Guided Interstitial Laser Therapy
}

\author{
S. Suprijanto ${ }^{1}$, M.W. Vogel ${ }^{2}$, F.M. $\operatorname{Vos}^{1}$, H.A. Vrooman ${ }^{2,3}$, and \\ A.M. Vossepoel ${ }^{1}$
}

1 Pattern Recognition Group, Department of Imaging Science and Technology Delft University of Technology 2628 CJ Delft The Netherlands

\{supri, frans, albert\}@ph.tn.tudelft.nl

2 Department of Medical Informatics, Erasmus University Medical Center

P.O. Box. 1738, 3000 DR Rotterdam, The Netherlands

3 Department of Radiology, Erasmus University Medical Center

P.O. Box. 1738, 3000 DR Rotterdam, The Netherlands

\{m.vogel,h.vrooman\}@erasmusmc.nl

\begin{abstract}
MR guided interstitial laser therapy can be used to monitor the extent of tumor tissue coagulation during thermal treatment based on a temperature map. In a non-stationary object that was influenced by respiratory motion, the temperature map may show errors due to incorrect spatial baseline images. Moreover, an unwanted phase shift due to object displacement contributes to errors in the temperature map, that must be suppressed. This paper describes a strategy which addresses the difficulties of MR guided interstitial laser therapy in the presence of respiratory motion. The multi-baseline images, supported by the displacement correction scheme, were used to improve the temporal resolution of a temperature map in the respiratory cycle. In the displacement correction scheme, the object coordinates that are provided by an active tracking coil were employed to support a matching strategy between the thermal and baseline images. To avoid errors in the temperature map due to the motion artifact, a detector of low quality images was proposed as part of the displacement correction scheme.
\end{abstract}

\section{Introduction}

The use of intense localized change in temperature is rapidly becoming an established surgical technique for destroying undesired tissue. Tissue ablation by percutaneous application of optical fibers and deposition of thermal energy via lasers, (called interstitial laser therapy), is a option for localized lesions, such as tumors, accessible via needle insertion. Dynamical temperature monitoring, with adequate spatial and temporal resolution could provide information about the extent of tissue coagulation. The goal is to avoid excess heating of surrounding healthy tissue and to ensure sufficient treatment of the tumor tissue.

MR Thermometry imaging is preferred for guided interstitial laser therapy, that is based on the relaxation time $\left(T_{1}\right)$, the diffusion coefficient (D) or proton resonance frequency (PRF) shift of tissue water. The excellent linearity and near independence with respect to tissue type, together with good temperature 
sensitivity, make MR Thermometry based on PRF shift the preferred choice. In this approach, the PRF shift due to temperature changes can be mapped using the MR phase images in gradient-echo imaging 13 .

In the absence of motion, MR thermometry based on phase imaging is quite accurate 113. Unfortunately, many clinical targets for tumor therapy however, especially in the abdomen, can not be held in stationary position due to respiration motion. In a non-stationary object, an error in the temperature map may occur due to incorrect spatial baseline images. Although a spatial transformation of the baseline image can be matched to the thermal image, an unwanted phase shift cannot be fully suppressed. Spatially varying field deviations can not be distinguished from temperature changes, and therefore leads to reduced thermal accuracy. A previous study showed that a reproducible phase image can be created, when scanning was done in the same position 4. To improve the temporal resolution of temperature measurement, an objective phase shift map must be determined from various scanning positions that occur in the respiratory cycle. Furthermore, multiple temperature maps can be interpolated to more accurately follow the temperature profile in time.

Another problem that must be considered is the error in the temperature maps due to the motion artifact. This artifact is caused by movement of an object during MR signal readout, yielding a low quality image. One approach to minimize the motion artifact is using a fast imaging technique. For MR thermometry based on phase imaging, the echo shifted gradient-echo imaging has been developed to increase the imaging speed with optimal temperature sensitivity [ $[$. Nevertheless, due to the variability of respiratory motion, occasionally a motion artifact occurs in an image.

In this paper, we describe a strategy to determine a temperature map based on a phase-shift map in the presence of respiratory motion. To recover a phaseshift map, we propose using multi-baseline images, supported by a displacement correction scheme. In this scheme, the object coordinates that are provided by an active tracking coil were employed to support a matching strategy between the thermal and baseline images. To avoid low quality images, a detector for sufficient image quality was proposed as part of the displacement correction scheme. For validation, the displacement correction scheme was applied to monitor temperature evolution during interstitial laser therapy in a liver model under respiratory motion simulation.

\section{Methods}

\subsection{Temperature Calculation Based on Objective Phase Shift Maps}

A complex MR image $I(\vec{r}, T)$ which is acquired by MRI at a certain temperature, is reconstructed by a Fourier transform of k-space data. Here, $\vec{r}$ and T denote the image coordinate and a specific temperature, respectively. $\Phi(\vec{r}, T)$ is a component of complex images that can be determined by taking $\arctan [I(\vec{r}, T)]$. The objective phase shift is formulated as follows [5]:

$$
\Psi(\overrightarrow{\Delta r}, \Delta T)=P U\left[\arctan \left[I(\vec{r}, T) \cdot I^{*}\left(\overrightarrow{r_{b l}}+\overrightarrow{\Delta r}, T_{o}\right)\right]+\Delta \phi(\overrightarrow{\Delta r})\right.
$$


where $I(\vec{r}, T), I\left(\overrightarrow{r_{b l}^{0}}, T_{o}\right)$ are respectively a thermal and a baseline image, $I^{*}$ is the complex conjugate of $I$. Phase unwrapping (PU) is used to recover the absolute phase shift in order to ensure that the phase shift is proportional with the large temperature variations in the therapeutic range. $\Delta \phi(\overrightarrow{\Delta r})$ is the bulk of an unwanted phase shift, that must be suppressed. Ideally, proper subtraction can be done when thermal and baseline images were scanned at exactly the same location. This condition can be expressed as $\vec{r}=\overrightarrow{r_{b l}^{0}}+\overrightarrow{\Delta r}$ and $\overrightarrow{\Delta r} \approx 0$. Furthermore, after an objective phase shift can be recovered, the temperature map in each sample time $\tau$ can be determined by taking the ratio between the current phase shift value and the temperature sensitivity $\left(\alpha \gamma B_{o} T_{E}\right)$ in $\mathrm{rad} /{ }^{\circ} \mathrm{C}$, that formulated as follow 13$]$ :

$$
\Delta T(\tau)=\frac{\Psi(\overrightarrow{\Delta r}, \Delta T, \tau)}{\alpha \gamma B_{o} T_{E}}
$$

where $\alpha$ is the temperature dependent water chemical shift, for which a value of $0.0101 \mathrm{ppm} /{ }^{\circ} \mathrm{C}$ is used in this work [3], $\gamma, B_{o}, T_{E}$ are respectively the gyromagnetic ratio, the magnetic field strength and the echo time.

\subsection{Displacement Correction Scheme}

Location Tags-Matching Strategy. The proper temperature maps can be determined when a thermal image matches with a baseline image. In a previous study, navigator echoes were employed for detection of 1-D object displacement, to support a matching strategy [5]. In this work, an active tracking coil was used to obtain the $3-\mathrm{D}$ coordinates, relative to the heat-source location. The active tracking coil was integrated into a power laser sheath which was introduced into the object. A custom receiver was used to detect a location information from the active tracking coil. In each scanning, the coordinates of location tags can be provided as additional information for MR images. The example of a series of intensity MR images and the location tag, that were acquired from a sample of porcine liver in a respiratory motion simulation, are shown in Fig. 1,
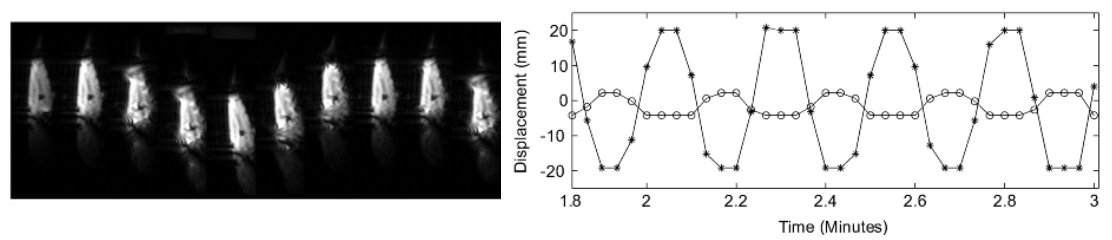

Fig. 1. (Left) Sequences of intensity images in respiratory motion simulation in one cycle, (Right) the location tags from the active tracking coil that are scanned during 1.2 minutes; 'o' and '*' are the location tag projections on the x-axis and $\mathrm{y}$-axis, respectively

The thermal image at a certain temperature $\mathrm{T}$ is given the notation $I(\vec{r}, T)$ and multi-baseline images are given the notation $\left.I\left(\overrightarrow{r_{b l}^{d}}, T_{o}\right)\right]$, where $b l=1,2, \ldots, n$ 
is a number of location tag for each multi-baseline image. The objective phase shift can be calculated when $I(\vec{r}, T)$ matches with one of $\left.I\left(\overrightarrow{r_{b l}}, T_{o}\right)\right]$, with minimized distance between $\vec{r}$ and one of $\left\{\overrightarrow{r_{1}^{o}}, \ldots, \overrightarrow{r_{n}^{0}}\right\}$, that is formulated as follows:

$$
\left|\Delta \overrightarrow{r_{b l}}\right|=\min \left\{\left|\Delta \overrightarrow{r_{1}}\right|, \ldots,\left|\Delta \overrightarrow{r_{n}}\right|\right\}
$$

where $\left|\Delta \overrightarrow{r_{1}}\right|=\left|\vec{r}-\overrightarrow{r_{1}^{o}}\right|=\left|\left(x-x_{1}^{o}\right),\left(y-y_{1}^{o}\right),\left(z-z_{1}^{o}\right)\right|$ and $\left|\Delta \overrightarrow{r_{n}}\right|=\left|\vec{r}-\overrightarrow{r_{n}^{o}}\right|=$ $\left|\left(x-x_{n}^{o}\right),\left(y-y_{n}^{o}\right),\left(z-z_{n}^{o}\right)\right|$.

The minimum distance value from one element $\left\{\left|\Delta \overrightarrow{r_{b}}\right|\right\}$ is related with the index $b l$. When the index $b l$ can be found, one of the multi-baseline images can be matched with a thermal image, allowing a temperature map to be calculated, based on objective phase subtraction, as formulated in Eq1.

Detection of Low Quality Images. In the series scanning, a location tag and an image are acquired, respectively, and updated in each scan. Due to the typical respiratory motion profile, it is possible that two images have a similar location tag, but different image quality. As we explained above, low quality images may occur due to the motion artifact acting as a filter that smears the temperature information in the MR phase images. Therefore, low quality images must be avoided. To support this task, image quality detection is proposed. The image quality can be determined from the degree of similarity between a grey value distributions of thermal and a baseline MR intensity image. As part of the displacement correction scheme, measurement of the image quality was done after the location tags matching criterion could be achieved. In this work, the Entropy Correlation Coefficient (ECC) [8], in formula

$$
E C C\left(S_{T}, S_{b l}\right)=\frac{2 I\left(S_{T}, S_{b l}\right)}{H\left(S_{T}\right)+H\left(S_{b l}\right)}
$$

was used, where $S_{T}$ and $S_{b l}$ represent the intensities of the thermal and the baseline images, $I\left(S_{T}, S_{b l}\right)$ and $H($.$) represent the mutual information of both images$ and the entropy of the image, respectively. The lowest value of the ECC that is yielding an unrealiable temperature map will be applied as a threshold value in the detector of low quality. The threshold value was determined experimentally.

\section{Materials and Results}

\subsection{Material}

All experiments were performed at a clinical MRI, GE CV/i $1.5 \mathrm{~T}$ scanner. Echoshifted gradient imaging was applied to allow formation of gradient echoes one or more repetition time (TR) periods after signal excitation. Thus echo timeTE $>$ TR is employed. A 3-inch surface coil was used as a receiver. As imaging parameters, $\mathrm{TR}=9 \mathrm{~ms}, \mathrm{TE}=17 \mathrm{~ms}, \mathrm{FOV}=250 \mathrm{~mm} \times 250 \mathrm{~mm}$, slice thickness $5 \mathrm{~mm}$ and flip angle $22^{\circ}$ are used. With these imaging parameters, acquisition time for each image is about 2 seconds. As heating source, a laser fiber (600 micrometer diameter) with a diffusing tip was inserted into the liver model. 
Laser irradiation (wavelength $1064 \mathrm{~nm}$ ) with a power of 10-25 Watt was applied as the heat-source. The laser sheath was then introduced into a sample of porcine liver. This was placed onto a device that simulated respiratory motion, with a motion profile as shown in Fig. 1

\subsection{Experiment Procedure}

Before the MR-guided interstitial laser was applied, the following steps were performed :

Step-I. The respiratory motion simulator was activated, after the heating and tracking devices had already been installed into the liver model. Without activated laser, pre-scanning was applied to acquire multi-baseline images were defined from location tags that simulated inspiration and expiration locations. Furthermore, a temperature map was calculated based on the location tags matching strategy. The criterion for minimum distance of $\left\{\left|\Delta \overrightarrow{r_{b l}}\right|\right\} \leq 1.0$ $\mathrm{mm}$ was selected. After that, a threshold value of ECC $\left(\Theta_{E C C}\right)$ was determined to eliminate the lowest quality images from temperature calculation, as follows:

- tabulation of relationship between background temperature and ECC values

- select a set of ECC values that have a relationship with the variation of the background temperature within a certain range

- calculation of mean $\left(\mu_{E C C}\right)$ and standard deviation $\left(\sigma_{E C C}\right)$ of the ECC from a set of ECC values, that was already selected

- The threshold value of $\Theta_{E C C}$ defined as $\mu_{E C C}-\sigma_{E C C}$

Step-II. In this step, the displacement correction scheme is ready to monitor temperature evolution for a liver model under interstitial laser therapy, in series scanning.

For reliable thermometry, the variation of the temperature should be within a range of $1^{\circ} \mathrm{C}$, a value that often can be achieved for immobile object 1 . From a previous study, a variation of temperature in the moving liver within range $\pm 3.5^{\circ} \mathrm{C}$ was reported after 6 minutes of laser irradiation [4]. Considering the ideal value and a tolerable value to the unwanted phase shift was not fully eliminated, the variation of the background temperature within $\pm 1.5^{\circ} \mathrm{C}$ was applied to select a set of ECC value.

\subsection{Experiment}

In the first experiment, the performance of the motion correction scheme was evaluated to calculate the background temperature in a liver model in the respiratory motion simulation, without activated laser. In step I, the baseline images were defined from images with location tags $\overrightarrow{r_{1}^{\circ}}=(-1.5 \mathrm{~mm}, 25.0 \mathrm{~mm}, 0.0 \mathrm{~mm})$ and $\overrightarrow{r_{2}^{o}}=(1.5 \mathrm{~mm},-25.0 \mathrm{~mm}, 0.0 \mathrm{~mm})$. The average temperature from 4 voxels, 5 $\mathrm{mm}$ from the laser tips position was used for evaluation. After that, pre-heating 
scanning with an effective duration of 2-3 minutes was done to determine $\Theta_{E C C}$. Then, tabulation of the relationship between background variation of temperature and the value of ECC was done. An example of the relationship between both parameters is shown in Fig. 2. Relationships between low value of ECC and the background variation of temperature beyond $\pm 1.5^{\circ}$ can be seen in this figure. Furthermore, after $\Theta_{E C C}$ was determined, the displacement correction scheme was ready to calculate background temperature from continuous scanning during 10 minutes. In Table-1, the standard deviation of background temperature
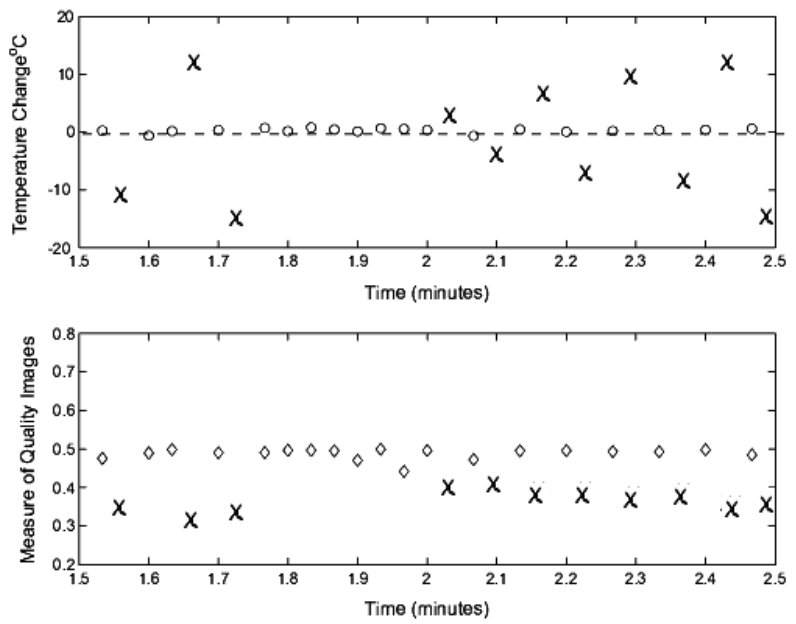

Fig. 2. Relation between the temperature variation and the value of ECC. The temperature maps that yield the background temperature beyond $\pm 1.5^{\circ} \mathrm{C}$ are marked by 'X', that relate to low values of ECC are also marked by 'X'

obtained from multi-baseline images supported by the location tags matching strategy alone, and by a full displacement correction scheme are presented. A number of thermal images can be included to calculate a temperature map from 300 images acquired during 10 minutes, also given in Table-1.

Table 1. Standard deviation of background temperature from continous scanning in duration 10 minutes, without activated laser

\begin{tabular}{|c|c|c|}
\hline Baseline Image & Location Tags Matching & Displacement correction scheme \\
\hline $\overrightarrow{r_{1}^{\circ}}$ & $4.9^{\circ} \mathrm{C}$ (92 images) & $1.1^{\circ} \mathrm{C}(62$ images $)$ \\
$\overrightarrow{r_{2}^{\sigma}}$ & $5.6^{\circ} \mathrm{C}$ (93 images) & $1.4^{\circ} \mathrm{C}(65$ images $)$ \\
Interpolating $\overrightarrow{r_{1}^{\mathrm{o}}}$ and $\overrightarrow{r_{2}^{\mathrm{d}}}$ & $6.2^{\circ} \mathrm{C}$ (185 images) & $1.2^{\circ} \mathrm{C}(127$ images $)$ \\
\hline
\end{tabular}

In the second experiment, the performance of the displacement correction scheme was evaluated for calculating the temperature evolution in the liver model in the respiratory motion simulation when laser power was activated. The range of respiratory motion was different from the first experiment. In step I, the 
baseline images were defined from two baseline images with location tags $\overrightarrow{r_{1}^{b}}=$ $(-4 \mathrm{~mm}, 25.0 \mathrm{~mm}, 0.0 \mathrm{~mm})$ and $\overrightarrow{r_{2}^{\delta}}=(4 \mathrm{~mm},-25.0 \mathrm{~mm}, 0.0 \mathrm{~mm})$. After that, preheating scanning during 4 minutes was applied to determine $\Theta_{E C C}$. In step II, after $\Theta_{E C C}$ was determined, the displacement correction scheme was ready to monitor temperature evolution. The scanning series was done during 12 minutes under laser irradiation to acquire thermal images.

The uncertainties of temperature evolution due to position difference among temperature maps were analyzed. From the $r_{2}$-temperature map, five types of exponential function were fitted to the temperature evolution on 5 location points (See Fig 3). Furthermore, the temperature evolution from the $r_{1}$-temperature map was calculated also from 5 location points. The standard deviation of the temperature uncertainties $\left(\sigma_{T r}\right)$ were used to measure the uncertainties of temperature evolution from the $r_{1}$-temperature map relative to the exponential function fitted to the temperature evolution from the $r_{2}$-temperature map. The results of $\sigma_{T r}$ from 5 location points are presented in Table 2 .

Finally, the area of iso-temperature was calculated from multiple 2-D temperature maps. The area evolution when $\mathrm{T}>43^{\circ} \mathrm{C}$ and $\mathrm{T}>50^{\circ} \mathrm{C}$ are presented in Fig-4.

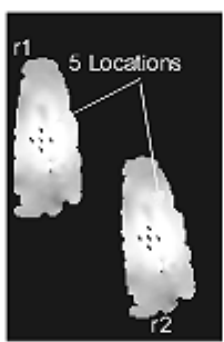

Temperaure maps on $r 1$ and $r 2$ positions

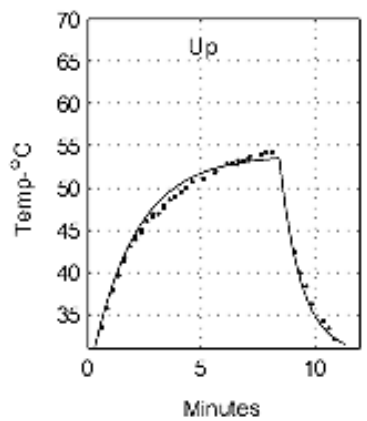

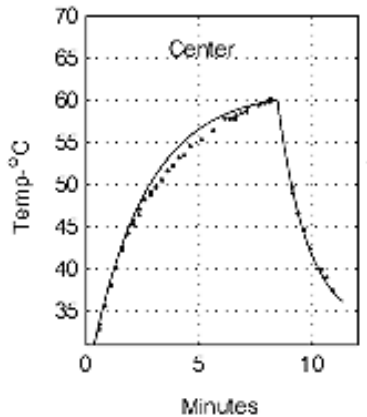
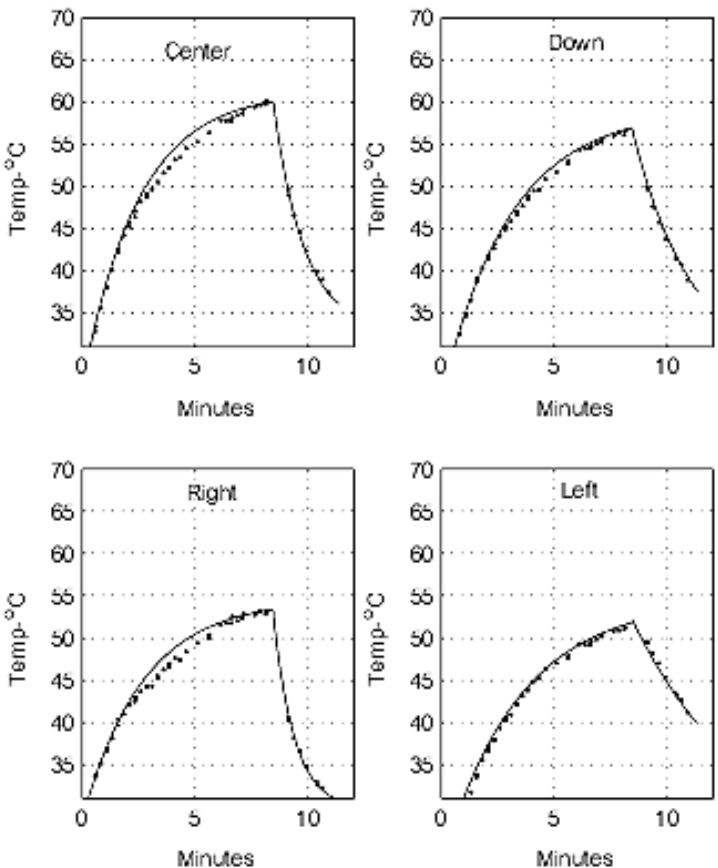

Fig. 3. The temperature evolution of the average of 4 voxels is measured at 5 location points in the temperature map. Five points were located at the center (i.e at laser tip), and $4 \mathrm{~mm}$ down, up, left and right relative to the center. The temperature evolution from the $r_{1}$-temperature map was marked by '.'. The exponential function that fitted to the temperature evolution from the $r_{2}$-temperature map was marked by '-'. 


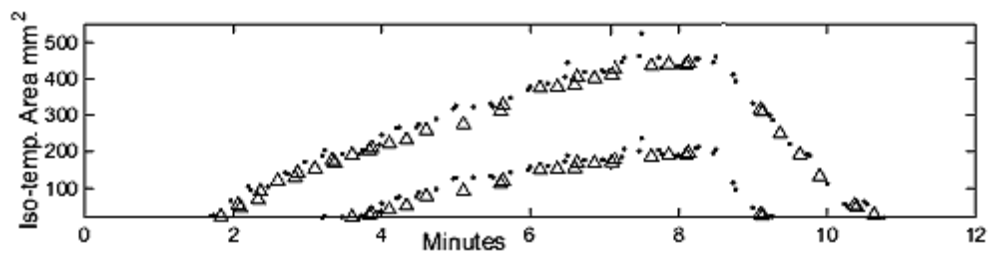

Fig. 4. The iso-temperature area $\left(\mathrm{mm}^{2}\right)$ from the temperature map for $T>43^{\circ} \mathrm{C}$ and $T>50^{\circ} C$; ' $\triangle$ ' and '.' resulted from the $r_{1}$ and $r_{2}$-temperature maps, respectively.

Table 2. The $\sigma_{T}$ between temperature evolution from the $r_{1}$-temperature map and the exponential functions that fitted to temperature evolution from the $r_{2}$-temperature map on 5 location points

\begin{tabular}{|c|c|c|c|c|c|}
\hline Location points & Center & Down & Up & Right & Left \\
\hline$\sigma_{T r}$ & $0.36^{\circ} \mathrm{C}$ & $0.29^{\circ} \mathrm{C}$ & $0.53^{\circ} \mathrm{C}$ & $0.42^{\circ} \mathrm{C}$ & $0.32^{\circ} \mathrm{C}$ \\
\hline
\end{tabular}

\section{Conclusions}

We have developed a displacement correction scheme for MR guided interstitial laser therapy, and this scheme was tested in a liver model under a simplified respiratory motion profile. When an object can be assumed rigid, the combination between a tracking coil employed for a location tags matching strategy on the one hand, and a detector of low quality images on the other hand are useful to support a multi-baseline image technique. When multiple temperature maps can be interpolated to more accurately follow the temperature profile, then the proposed scheme will improve the temporal resolution of tissue-coagulation monitoring during interstitial laser therapy.

Acknowledgements. This research is part of the MISIT (Minimally Invasive Surgery and Interventional Techniques) programme of the Delft Interfaculty Research Center on Medical Engineering (DIOC-9) and the authors thank to C. DuMoulin and R. Darrow for the active tracking system.

\section{References}

1. B.Quesson, J.A.de Zwart, C.T.W. Moonen, Magnetic resonance temperature imaging for guidance of thermotherapy, J.Magn Reson Im 2000; 12: 525-533 
2. Wlodarcyk W,Boroschewski R, Hentshel M, at.al. Comparison of four magnetic resonance methods for mapping small temperature change, Physics Medical Biology Vol. 44, pp. 607-624, 1999.

3. M.W. Vogel, Suprijanto, F.M. Vos, H.A. Vrooman,A.M. Vossepoel,P.M.T. Pattynama. Towards Motion-Robust Magnetic Resonance Thermometry, MICCAI 2001 Proceedings:LNCS Vol 2208, Springer-Verlag ,Berlin pp.401-408, 2001

4. Heisterkamp J, Matheijssen NA, van Hillegersberg R et al. Accuracy of MR phase mapping for temperature monitoring during interstitial laser coagulation (ILC) in the liver at rest and simulated respiration, Magn Reson Med 1999; 41: 919-925

5. J.A. de Zwart, F.C. Vimeux , J.Palussière, R.Salomir 1, B. Quesson, C.Delalande, C.T.W. Moonen. On-line correction and visualization of motion during MRIcontrolled hyperthermia, Magnetic Resonance in Medicine Volume 45, Issue 1, 2001. Pages: 128-137

6. Dennis C. Ghiglia. Mark D. Pritt. Two-Dimensional Phase Unwrapping, Theory, Algorithm and Software, John Willey and Sons, 1998

7. Muralidharan V. and Christophi C. Intestitial laser Thermotheraphy in The Treatment of Colorectal liver Metastases, Journal of Surgical Oncology, No. 76, pp.73-81, 2001

8. J.V. Hajnal, D.L.G. Hill, D.J. Hawkes. Medical Image Registration, CRC Press LCC 2001 